\title{
Solving Fixed-Point Problems with Inequality and Equality Constraints via a Non-Interior Point Homotopy Path-Following Method
}

\author{
Menglong Su ${ }^{1,2}$ and Yufeng Shang ${ }^{3}$ \\ ${ }^{1}$ School of Mathematics, Luoyang Normal University, Luoyang 471934, China \\ ${ }^{2}$ Key Laboratory of Symbolic Computation and Knowledge Engineering of Ministry of Education, Jilin University, \\ Changchun 130012, China \\ ${ }^{3}$ Section of Mathematics, Aviation University of Air Force, Changchun 130022, China
}

Correspondence should be addressed to Menglong Su; sumenglongjlu@163.com

Received 10 July 2017; Revised 1 October 2017; Accepted 15 October 2017; Published 16 November 2017

Academic Editor: Rosana Rodríguez-López

Copyright $\odot 2017$ Menglong Su and Yufeng Shang. This is an open access article distributed under the Creative Commons Attribution License, which permits unrestricted use, distribution, and reproduction in any medium, provided the original work is properly cited.

In recent years, fixed-point theorems have attracted increasing attention and have been widely investigated by many authors. Moreover, determining a fixed point has become an interesting topic. In this paper, we provide a constructive proof of the general Brouwer fixed-point theorem and then obtain the existence of a smooth path which connects a given point to the fixed point. We also present a non-interior point homotopy algorithm for solving fixed-point problems on a class of nonconvex sets by numerically tricking this homotopy path.

\section{Introduction}

In recent years, fixed-point theorems have attracted increasing attention and have been widely investigated by many authors (e.g., [1-4] and the references therein) because these theorems play important roles in mechanics, physics, differential equations, and so on. The determination of a constructive proof of the fixed-point theorem and therefore finding a fixed point became an attractive topic. The homotopy method, as a globally convergent algorithm, is a powerful tool in handling fixed-point problems (e.g., [5-10] and the references therein). The general Brouwer fixed-point theorem states that if a bounded closed subset in $R^{n}$ is diffeomorphic to the closed unit ball, then any continuous self-mapping in it has a fixed point. However, the abovementioned results generally require certain convexity assumptions; thus, the traditional homotopy method cannot be used to handle the general Brouwer fixed-point theorem. Until 1996, Yu and Lin [11] combined the ideas of interior point methods and homotopy methods to propose a homotopy interior pathfollowing method (see [12-17] for more details) that provides a constructive proof of the general Brouwer fixed-point theorem on a class of nonconvex subsets, without constructing a homeomorphism for transforming the bounded closed set to the closed unit ball. In $[18,19]$, the authors furthermore extended the results in [11] to more general nonconvex sets with inequality and equality constraint functions by replacing the gradient mappings with the newly introduced $C^{2}$ mappings.

The expansion of the scope of initial point selection to improve the computational efficiency of an algorithm is an important research area. In [20], we applied appropriate perturbations to the constraint functions and developed a new homotopy method to expand the scope of initial point selection, but involving the inequality constraint cases only. In [21], using similar perturbations to the inequality constraints in [20], we mainly extended the results in [18] to unbounded cases by providing a set of unbounded conditions. It should be pointed out that the results in $[18,19]$ excluded the initial point selection; in addition, the researchers excluded the equality constraint cases, although the results in $[20,21] \mathrm{ex}-$ panded the scope of initial point selection. It is difficult to 
construct appropriate perturbations and to guarantee the regularity of the homotopy matrix for the existence of the equality constraints. To overcome the difficulties mentioned above, we apply new perturbations to the equality constraints and construct a new homotopy matrix to guarantee its regularity. Therefore, we develop a non-interior point homotopy path-following method for solving fixed-point problems with inequality and equality constraints. We can select initial points easily and thus considerably improve the computational efficiency of the algorithm by using the new approach.

This paper is organized as follows. Section 2 introduces two parameters and constructs appropriate perturbations to the constraint functions to develop a non-interior point homotopy path-following method for solving fixed-point problems with equality and inequality constraints. Section 3 presents several experimental examples to illustrate the results in this paper.

\section{Main Results}

In this section, we use the following notations: $x \in R^{n}$, $y \in R^{m}, z \in R^{l}, g(x)=\left(g_{1}(x), \ldots, g_{m}(x)\right)^{T}, h(x)=$ $\left(h_{1}(x), \ldots, h_{m}(x)\right)^{T}, \nabla g(x)=\left(\nabla g_{1}(x), \ldots, \nabla g_{m}(x)\right) \quad \epsilon$ $R^{n \times m}, \nabla h(x)=\left(\nabla h_{1}(x), \ldots, \nabla h_{l}(x)\right) \in R^{n \times l}, \alpha(x)=$ $\left(\alpha_{1}(x), \ldots, \alpha_{m}(x)\right) \in R^{n \times m}, \beta(x)=\left(\beta_{1}(x), \ldots, \beta_{l}(x)\right) \in R^{n \times l}$, $X=\left\{x \in R^{n}: g(x) \leq 0, h(x)=0\right\}, X^{0}=\left\{x \in R^{n}: g(x) \leq\right.$ $0, h(x)=0\}, R_{+}^{m}=\left\{x \in R^{m}: x \geq 0\right\}, R_{++}^{m}=\left\{x \in R^{m}: x>0\right\}$, and $B(x)=\left\{i \in\{1, \ldots, m\}: g_{i}(x)=0\right\}$.

In [18], we extended the results in [11] to more general sets under the following assumptions:

$\left(\mathrm{A}_{1}\right) X^{0}$ is nonempty and $X$ is bounded.

$\left(\mathrm{A}_{2}\right)$ For any $x \in X$, if

$$
\sum_{i \in B(x)}\left(u_{i} \nabla g_{i}(x)+y_{i} \alpha_{i}(x)\right)+\sum_{j=1}^{l} z_{j} \beta_{j}(x)=0
$$

$$
u_{i} \geq 0, \quad y_{i} \geq 0, z_{j} \in R^{1}
$$

then $u_{i}=0, y_{i}=0, \forall i \in B(x)$, and $z_{j}=0, j=1, \ldots, l$.

$\left(\mathrm{A}_{3}\right)$ The weak normal cone condition of $X$ : for any $x \in X$, we have

$$
\begin{aligned}
& \left\{x+\sum_{i \in B(x)} y_{i} \alpha_{i}(x)+\beta(x) z: u_{i} \geq 0, i \in B(x), z\right. \\
& \left.\in R^{l}\right\} \cap X=\{x\} .
\end{aligned}
$$

$\left(\mathrm{A}_{4}\right)$ For any $x \in X, \nabla h(x)$ is of full column rank and $\nabla h(x)^{T} \beta(x)$ is nonsingular.
In this study, we introduce the following parameters to construct appropriate perturbations to the constraint functions:

$$
\begin{aligned}
& \gamma_{i}= \begin{cases}2 g_{i}\left(x^{(0)}\right), & g_{i}\left(x^{(0)}\right)>0, \\
1, & g_{i}\left(x^{(0)}\right)=0, \\
0, & g_{i}\left(x^{(0)}\right)<0,\end{cases} \\
& \theta_{j}= \begin{cases}1, & h_{j}\left(x^{(0)}\right) \neq 0, \ldots, m, \\
0, & h_{j}\left(x^{(0)}\right)=0,\end{cases}
\end{aligned}
$$

where $x^{(0)}$ is an arbitrarily given point. Then, set $\Upsilon=$ $\operatorname{diag}\left(\gamma_{1}, \ldots, \gamma_{m}\right), \Theta=\operatorname{diag}\left(\theta_{1}, \ldots, \theta_{l}\right), e_{m}=(1, \ldots, 1)^{T} \in$ $R^{m}, X(\lambda)=\left\{x \in R^{n}: g(x)-\lambda \Upsilon\left(g\left(x^{(0)}\right)+e_{m}\right) \leq 0, h(x)-\right.$ $\left.\lambda \Theta h\left(x^{(0)}\right)=0\right\}, X^{0}(\lambda)=\left\{x \in R^{n}: g(x)-\lambda \Upsilon\left(g\left(x^{(0)}\right)+e_{m}\right)<\right.$ $\left.0, h(x)-\lambda \Theta h\left(x^{(0)}\right)=0\right\}$, and $I(x, \lambda)=\{i \in\{1, \ldots, m\}$ : $\left.g_{i}(x)-\lambda \gamma_{i}\left(g_{i}\left(x^{(0)}\right)+1\right)=0\right\}$.

To solve fixed-point problems in more general nonconvex sets, we also introduce the continuous mappings $\xi(x, u)=\left(\xi_{1}\left(x, u_{1}\right), \ldots, \xi_{m}\left(x, u_{m}\right)\right) \in R^{n \times m}$ and $\eta(x, v)=$ $\left(\eta_{1}\left(x, v_{1}\right), \ldots, \eta_{l}\left(x, v_{l}\right)\right) \in R^{n \times l}$ which satisfy the following conditions:

$\left(\mathrm{C}_{1}\right) X^{0}(\lambda)$ is nonempty and $X(\lambda)$ is bounded.

$\left(\mathrm{C}_{2}\right) \xi_{i}(x, 0)=0, i=1, \ldots, m, \eta_{j}(x, 0)=0, j=1, \ldots, l$; besides, for any $x \in X(\lambda)$, if $\|(y, z, u, v)\| \rightarrow \infty$, then

$$
\| \sum_{i \in I(x, \lambda)}\left(y_{i} \nabla g_{i}(x)+\xi_{i}\left(x, u_{i}\right)\right)+\nabla h(x) z
$$

$$
+\sum_{j=1}^{l} \eta\left(x, v_{j}\right) \| \rightarrow \infty
$$

$\left(\mathrm{C}_{3}\right)$ For any $x \in X(\lambda)$, if

$$
\begin{aligned}
& \sum_{i \in I(x, \lambda)}\left(y_{i} \nabla g_{i}(x)+\xi_{i}\left(x, u_{i}\right)\right)+\nabla h(x) z+\sum_{j=1}^{l} \eta\left(x, v_{j}\right) \\
& \quad=0, \quad y_{i} \geq 0, \quad u_{i} \geq 0,
\end{aligned}
$$

then $y_{i}=0, u_{i}=0, \forall i \in I(x, \lambda), z=0, v_{j}=0, j=1, \ldots, l$. Besides, the matrix $\nabla h(x)^{T} \nabla_{z}\left(\sum_{j=1}^{l} \eta_{j}\left(x, z_{j}\right)\right)$ is nonsingular. 
$\left(\mathrm{C}_{4}\right)$ When $\lambda=0,1$, for any $x \in X(\lambda)$, we have

$$
\begin{aligned}
& \left\{x+\sum_{i \in I(x, \lambda)} \xi_{i}\left(x, u_{i}\right)+\sum_{j=1}^{l} \eta\left(x, v_{j}\right): u_{i} \geq 0 \text { for } i\right. \\
& \left.\in I(x, \lambda), v_{j} \in R^{1}\right\} \cap X(\lambda)=\{x\} .
\end{aligned}
$$

From the geometric perspective in $R^{2}$, we explain that the results in [18] are extended to more general nonconvex sets. Set $X_{1}=\left\{x+\sum_{i \in B(x)} y_{i} \alpha_{i}(x)+\beta(x) z\right\}$; note that $\sum_{i \in B(x)} y_{i} \alpha_{i}(x)+\beta(x) z$ is the linear combinations of $\alpha_{i}(x) \in R^{n}$, $i=1, \ldots, m$, and $\beta_{j}(x) \in R^{n}, j=1, \ldots, l$, and the set $X_{1}$ is not a bending cone surrounded by several radials or beelines; thus, many nonconvex sets cannot satisfy assumption $\left(\mathrm{A}_{3}\right)$ in [18]. However, set $X_{2}=\left\{x+\sum_{i \in I(x, 0)} \xi_{i}\left(x, u_{i}\right)+\sum_{j=1}^{l} \eta_{j}\left(x, v_{j}\right)\right\}$. Note that $\alpha_{i}(x) \in R^{n}, i=1, \ldots, m$, and $\beta_{j}(x) \in R^{n}, j=$ $1, \ldots, l$, are the special cases of $\xi_{i}\left(x, u_{i}\right) \in R^{n}, i=1, \ldots, m$, and $\eta_{j}\left(x, v_{j}\right) \in R^{n}, j=1, \ldots, l$; in many cases, $X_{2}$ may be a bending cone surrounded by several curves because $\xi_{i}\left(x, u_{i}\right)$, $i=1, \ldots, m$, and $\eta_{j}\left(x, v_{j}\right), j=1, \ldots, l$, are arbitrary functions of $u_{i}, i=1, \ldots, m, v_{j}, j=1, \ldots, l$. This point enables many nonconvex sets to not satisfy assumption $\left(\mathrm{A}_{3}\right)$ but to satisfy assumption $\left(\mathrm{C}_{4}\right)$.

To solve fixed-point problems, we construct the following new homotopy equation:

$$
\begin{aligned}
& H\left(P, P^{(0)}, \lambda\right) \\
& =\left(\begin{array}{c}
(1-\lambda)\left(x-\Phi(x)+\sum_{i=1}^{m}(1-\lambda) \lambda \nabla g_{i}(x) y_{i}\right)+\sum_{i=1}^{m} \xi_{i}\left(x,(1-\lambda) y_{i}\right)+(1-\lambda) \lambda(\nabla h(x) z+\alpha)+\sum_{j=1}^{l} \eta_{j}\left(x, z_{j}\right)+\lambda\left(x-x^{(0)}\right) \\
h(x)-\lambda \Theta h\left(x^{(0)}\right) \\
Y\left(g(x)-\lambda \Upsilon\left(g\left(x^{(0)}\right)+e_{m}\right)\right)-\lambda Y^{(0)}\left(g\left(x^{(0)}\right)-\Upsilon\left(g\left(x^{(0)}\right)+e_{m}\right)\right)
\end{array}\right)
\end{aligned}
$$

$=0$,

where $P=(x, y, z) \in R^{n+m+l}, P^{(0)}=\left(x^{(0)}, y^{(0)}, z^{(0)}\right) \in X^{0}(1) \times$ $R_{++}^{m} \times R^{l}, \alpha \in R^{n}, Y=\operatorname{diag}(y)$, and $Y^{(0)}=\operatorname{diag}\left(y^{(0)}\right)$.

We rewrite $H\left(P, P^{(0)}, \lambda\right)$ as $H_{P^{(0)}}(P, \lambda)$ for a given $P^{(0)}$. The zero-point set of $H_{P^{(0)}}$ is

$$
\begin{aligned}
& H_{P^{(0)}}^{-1}(0) \\
& \quad=\left\{(P, \lambda) \in R^{n} \times R_{+}^{m} \times R^{l} \times(0,1]: H_{P^{(0)}}(P, \lambda)=0\right\} .
\end{aligned}
$$

Lemmas 1-4 will be used in the proof of our main results.

Lemma 1 (see [22]). Let $\Phi: R^{n+1} \rightarrow R^{n}$ be a $C^{1}$ map and 0 a regular value of $\Phi$. Then, $\Phi^{-1}(0)$ is a $C^{1}$ manifold of dimension 1 .

Lemma 2 (see [22]). A $C^{1}$ manifold of dimension 1 is diffeomorphic to a loop or an interval.

Lemma 3 (transversality theorem). Let $Q, N$, and $P$ be smooth manifolds with dimensions $q, m$, and $\widetilde{p}$, respectively. Let $W \subset P$ be a submanifold of codimension $p$ (i.e., the $\widetilde{p}=p+$ dimension of $W)$. Consider a smooth map $\Phi: Q \times N \rightarrow P$. If $\Phi$ is transversal to $W$, then, for almost all $a \in Q, \Phi_{a}(\cdot)=$ $\Phi(a, \cdot): N \rightarrow P$ is transversal to $W$. Recall that a smooth map $h: N \rightarrow P$ is transversal to $W$ if

$$
\{\operatorname{Range}(\operatorname{Dh}(x))\}+\left\{T_{y} W\right\}=T_{y} P
$$

$$
\text { whenever } y=h(x) \in W \text {, }
$$

where $D h$ is the Jacobi matrix of $h$ and $T_{y} W$ and $T_{y} P$ denote the tangent spaces of $W$ and $P$ at $y$, respectively.
In this paper, $W=\{0\}$; thus, Lemma 3 corresponds to Lemma 4.

Lemma 4 (parameterized Sard's theorem). Let $V \subset R^{n}$ and $U \subset R^{m}$ be open sets and $\Phi: V \times U \rightarrow R^{k}$ be a $C^{r}$ map, where $r>\max \{0, m-k\}$. If $0 \in R^{k}$ is a regular value of $\Phi$, then, for almost all $a \in V, 0$ is a regular value of $\Phi_{a} \equiv \Phi(a, \cdot)$.

Lemma 5. Let $H$ be defined as in (7); let $g_{i}(x), i=1, \ldots, m$, and $h_{j}(x), j=1, \ldots, l$, be $C^{3}$ functions; let assumptions $\left(C_{1}\right)-\left(C_{4}\right)$ hold; and let $\xi_{i}\left(x, u_{i}\right) \in R^{n}, i=1, \ldots, m$, and $\eta_{j}\left(x, v_{j}\right) \in R^{n}, j=1, \ldots, l$, be $C^{2}$ functions. Then, for almost all $x^{0} \in X^{0}(1), 0$ is a regular value of map $H_{P^{(0)}}$ : $R^{n} \times R_{+}^{m} \times R^{l} \times(0,1] \rightarrow R^{n+m+l}$, and $H_{P^{(0)}}^{-1}(0)$ consists of some smooth curves. Among them, a smooth curve, denoted by $\Gamma_{P^{(0)}}$, starts from $\left(P^{(0)}, 1\right)$.

Proof. We denote $H\left(P, P^{(0)}, \lambda\right)$ by $\bar{H}\left(P, P^{(0)}, \alpha, \lambda\right)$ when $\alpha$ is considered as a variable. Let the Jacobian matrix of $\bar{H}\left(P, P^{(0)}, \alpha, \lambda\right)$ be denoted by $D \bar{H}\left(P, P^{(0)}, \alpha, \lambda\right)$. For any $\lambda \epsilon$ $(0,1]$,

$$
\frac{\partial \bar{H}\left(P, P^{(0)}, \alpha, \lambda\right)}{\partial(x, \alpha, y)}
$$

$$
=\left(\begin{array}{ccc}
A & \lambda(1-\lambda) I & B \\
\nabla h(x)^{T} & 0 & 0 \\
Y \nabla g(x)^{T} & 0 & \operatorname{diag}\left(g(x)-\lambda \Upsilon\left(g\left(x^{(0)}\right)+e_{m}\right)\right)
\end{array}\right),
$$


where

$$
\begin{aligned}
A= & (1-\lambda)\left(I-\nabla \Phi(x)+\sum_{i=1}^{m}(1-\lambda) \lambda \nabla^{2} g_{i}(x) y_{i}\right) \\
& +\sum_{i=1}^{m} \nabla_{x} \xi_{i}\left(x,(1-\lambda) y_{i}\right)+(1-\lambda) \lambda \nabla^{2} h(x) z \\
& +\sum_{j=1}^{l} \nabla_{x} \eta_{j}\left(x, z_{j}\right)+\lambda I, \\
B= & (1-\lambda)^{2} \lambda \nabla g(x)^{T} \\
& +(1-\lambda) \sum_{i=1}^{m}\left(\nabla_{y_{i}} \xi_{i}\left(x,(1-\lambda) y_{i}\right)\right)^{T} .
\end{aligned}
$$

Because $\nabla h(x)^{T}$ is a matrix of full row rank, $\partial \bar{H}\left(P, P^{(0)}, \alpha, \lambda\right) / \partial(x, \alpha, y)$ is of full row rank. Therefore, $D \bar{H}\left(P, P^{(0)}, \alpha, \lambda\right)$ is also of full row rank, and 0 is a regular value of $\bar{H}\left(P, P^{(0)}, \alpha, \lambda\right)$. By Lemma 4 , for almost all $P^{(0)} \in X^{0}(1) \times R_{++}^{m} \times R^{l}$, we obtain that 0 is a regular value of map $H_{P^{(0)}}: R^{n} \times R_{+}^{m} \times R^{l} \times(0,1] \rightarrow R^{n+m+l}$. By Lemma $1, H_{P^{(0)}}^{-1}(0)$ consists of several smooth curves. Because $H_{P^{(0)}}\left(P^{(0)}, 1\right)=0$, then a $C^{1}$ curve $(P(s), \lambda(s))$ of dimension 1 , denoted by $\Gamma_{P^{(0)}}$, starts from $\left(P^{(0)}, 1\right)$.

Lemma 6. Let $H$ be defined as in (7); let $g_{i}(x), i=1, \ldots, m$, and $h_{j}(x), j=1, \ldots, l$, be $C^{3}$ functions; let assumptions $\left(C_{1}\right)-\left(C_{4}\right)$ hold; and let $\xi_{i}\left(x, u_{i}\right) \in R^{n}, i=1, \ldots, m$, and $\eta_{j}\left(x, v_{j}\right) \in R^{n}, j=1, \ldots, l$, be $C^{2}$ functions. Then, for almost all $x^{0} \in X^{0}(1), \Gamma_{P^{(0)}}$ is a bounded curve.

Proof. Assume that $\Gamma_{P^{(0)}}$ is an unbounded curve. Then, there exists a sequence of points $\left\{\left(P^{(k)}, \lambda_{k}\right)\right\} \subset \Gamma_{P^{(0)}}$ such that $\left\|P^{(k)}, \lambda_{k}\right\| \rightarrow \infty$. Because $X(\lambda)$ and $(0,1]$ are bounded, hence there exists a subsequence of points (denoted also by $\left.\left\{\left(P^{(k)}, \lambda_{k}\right)\right\}\right)$ such that $x^{(k)} \rightarrow x^{*},\left\|\left(y^{(k)}, z^{(k)}\right)\right\| \rightarrow \infty$, and $\lambda_{k} \rightarrow \lambda^{*}$ as $k \rightarrow \infty$. From the homotopy equation (7), we obtain

$$
\begin{aligned}
& \left(1-\lambda_{k}\right)\left(x^{(k)}-\Phi\left(x^{(k)}\right)\right. \\
& \left.+\sum_{i=1}^{m}\left(1-\lambda_{k}\right) \lambda_{k} \nabla g_{i}\left(x^{(k)}\right) y_{i}^{(k)}\right) \\
& +\sum_{i=1}^{m} \xi_{i}\left(x^{(k)},\left(1-\lambda_{k}\right) y_{i}^{(k)}\right)+\left(1-\lambda_{k}\right)
\end{aligned}
$$

$$
\begin{aligned}
& \cdot \lambda_{k}\left(\nabla h\left(x^{(k)}\right) z^{(k)}+\alpha\right)+\sum_{j=1}^{l} \eta_{j}\left(x^{(k)}, z_{j}^{(k)}\right) \\
& +\lambda_{k}\left(x^{(k)}-x^{(0)}\right)=0, \\
& h\left(x^{(k)}\right)-\lambda_{k} \Theta h\left(x^{(0)}\right)=0, \\
& Y^{(k)}\left(g\left(x^{(k)}\right)-\lambda_{k} \Upsilon\left(g\left(x^{(0)}\right)+e_{m}\right)\right) \\
& \quad-\lambda_{k} Y^{(0)}\left(g\left(x^{(0)}\right)-\Upsilon\left(g\left(x^{(0)}\right)+e_{m}\right)\right)=0 .
\end{aligned}
$$

Let

$$
\begin{aligned}
& I\left(x^{*}\right)=\left\{i \in\{1, \ldots, m\}: \lim _{k \rightarrow \infty} y_{i}^{(k)}=\infty\right\}, \\
& J\left(x^{*}\right)=\left\{j \in\{1, \ldots, l\}: \lim _{k \rightarrow \infty} z_{j}^{(k)}=\infty\right\} .
\end{aligned}
$$

If $J\left(x^{*}\right) \neq \emptyset$, from (12), one obtains

$$
\begin{aligned}
& \left(1-\lambda_{k}\right)\left(x^{(k)}-\Phi\left(x^{(k)}\right)\right) \\
& \quad+\sum_{i \notin I\left(x^{*}\right)}\left(1-\lambda_{k}\right)^{2} \lambda_{k} \nabla g_{i}\left(x^{(k)}\right) y_{i}^{(k)} \\
& \quad+\sum_{i \notin I\left(x^{*}\right)} \xi_{i}\left(x^{(k)},\left(1-\lambda_{k}\right) y_{i}^{(k)}\right)+\lambda_{k}\left(x^{(k)}-x^{(0)}\right) \\
& +\left(1-\lambda_{k}\right) \lambda_{k}\left(\nabla h\left(x^{(k)}\right) z^{(k)}+\alpha\right) \\
& \quad+\sum_{j=1}^{l} \eta_{j}\left(x^{(k)}, z_{j}^{(k)}\right) \\
& +\sum_{i \in I\left(x^{*}\right)}\left(1-\lambda_{k}\right)^{2} \lambda_{k} \nabla g_{i}\left(x^{(k)}\right) y_{i}^{(k)} \\
& \quad+\sum_{i \in I\left(x^{*}\right)} \xi_{i}\left(x^{(k)},\left(1-\lambda_{k}\right) y_{i}^{(k)}\right)=0 .
\end{aligned}
$$

The sixth to ninth parts in the left-hand side of (16) tend to infinity as $k \rightarrow \infty$, but the other five parts are bounded, which is impossible. Therefore, the projection of the smooth curve $\Gamma_{P^{(0)}}$ onto the $z$-plane is also bounded. Now, we can assume that $z^{(k)} \rightarrow z^{*}$. Simultaneously, $I\left(x^{*}\right) \neq \emptyset$.

If case (c) holds, then there exists a sequence of points $\left\{\left(P^{(k)}, \lambda_{k}\right)\right\} \subset \Gamma_{P^{(0)}}$ such that $\left\|\left(P^{(k)}, \lambda_{k}\right)\right\| \rightarrow \infty$. Because $X(\lambda)$ and $(0,1]$ are bounded, hence there exists a subsequence of points (denoted also by $\left\{\left(P^{(k)}, \lambda_{k}\right)\right\}$ ) such that $x^{(k)} \rightarrow x^{*}$, $\left\|y^{(k)}\right\| \rightarrow \infty, z^{(k)} \rightarrow z^{*}$, and $\lambda_{k} \rightarrow \lambda_{*}$ as $k \rightarrow \infty$. From (14), we obtain

$$
\begin{array}{r}
g_{i}\left(x^{(k)}\right)-\lambda_{k} \gamma_{i}\left(g_{i}\left(x^{(0)}\right)+1\right) \\
=\lambda_{k}\left(y_{i}^{(k)}\right)^{-1} y_{i}^{(0)}\left(g_{i}\left(x^{(0)}\right)-\gamma_{i}\left(g_{i}\left(x^{(0)}\right)+1\right)\right), \\
i=1, \ldots, m .
\end{array}
$$


When $\lambda^{*}>0$, the index set is

$$
I\left(x^{*}, \lambda^{*}\right)=\left\{i \in\{1, \ldots, m\}: \lim _{k \rightarrow \infty} y_{i}^{(k)}=\infty\right\} .
$$

When $\lambda^{*}=0$, the index set is

$$
\begin{aligned}
I_{0}\left(x^{*}, 0\right) & =\left\{i \in\{1, \ldots, m\}: \lim _{k \rightarrow \infty} y_{i}^{(k)}=\infty\right\} \\
& \subset I\left(x^{*}, 0\right) .
\end{aligned}
$$

(1) If $\lambda^{*}=1$, from (12), we obtain

$$
\begin{aligned}
x^{(k)}- & x^{(0)}+\sum_{i \in I\left(x^{*}, 1\right)}\left(1-\lambda_{k}\right)^{2} \lambda_{k} \nabla g_{i}\left(x^{(k)}\right) y_{i}^{(k)} \\
& +\sum_{i \in I\left(x^{*}, 1\right)} \xi_{i}\left(x^{(k)},\left(1-\lambda_{k}\right) y_{i}^{(k)}\right) \\
& +\left(1-\lambda_{k}\right) \lambda_{k}\left(\nabla h\left(x^{(k)}\right) z^{(k)}+\alpha\right) \\
& +\sum_{j=1}^{l} \eta_{j}\left(x^{(k)}, z_{j}^{(k)}\right) \\
= & -\sum_{i \notin I\left(x^{*}, 1\right)}\left(1-\lambda_{k}\right)^{2} \lambda_{k} \nabla g_{i}\left(x^{(k)}\right) y_{i}^{(k)} \\
& -\sum_{i \notin I\left(x^{*}, 1\right)} \xi_{i}\left(x^{(k)},\left(1-\lambda_{k}\right) y_{i}^{(k)}\right) \\
& +\left(1-\lambda_{k}\right)\left(\Phi\left(x^{(k)}\right)-x^{(0)}\right) .
\end{aligned}
$$

By assumptions $\left(\mathrm{C}_{2}\right),\left(\mathrm{C}_{3}\right)$, and $(20)$, we obtain

$$
\lim _{k \rightarrow \infty}\left(1-\lambda_{k}\right) y_{i}^{(k)}=y_{i}^{*}
$$

where $y_{i}^{*} \geq 0$. Therefore, from (20) and (21), we obtain

$$
x^{*}+\sum_{i \in I\left(x^{*}, 1\right)} \xi_{i}\left(x^{*}, y_{i}^{*}\right)+\sum_{j=1}^{l} \eta_{j}\left(x^{(k)}, z_{j}^{*}\right)=x^{(0)},
$$

which contradicts assumption $\left(\mathrm{C}_{4}\right)$.

(2) If $0<\lambda^{*}<1$, from (12), we obtain

$$
\begin{gathered}
\sum_{i \in I\left(x^{*}, \lambda^{*}\right)}\left(1-\lambda_{k}\right)^{2} \lambda_{k} \nabla g_{i}\left(x^{(k)}\right) y_{i}^{(k)} \\
+\sum_{i \in I\left(x^{*}, \lambda^{*}\right)} \xi_{i}\left(x^{(k)},\left(1-\lambda_{k}\right) y_{i}^{(k)}\right) \\
=-\sum_{i \notin I\left(x^{*}, \lambda^{*}\right)}\left(1-\lambda_{k}\right)^{2} \lambda_{k} \nabla g_{i}\left(x^{(k)}\right) y_{i}^{(k)} \\
\quad-\sum_{i \notin I\left(x^{*}, \lambda^{*}\right)} \xi_{i}\left(x^{(k)},\left(1-\lambda_{k}\right) y_{i}^{(k)}\right) \\
-\left(1-\lambda_{k}\right) \lambda_{k}\left(\nabla h\left(x^{(k)}\right) z^{(k)}+\alpha\right)
\end{gathered}
$$

$$
\begin{aligned}
& -\sum_{j=1}^{l} \eta_{j}\left(x^{(k)}, z_{j}^{(k)}\right)-\left(1-\lambda_{k}\right)\left(x^{(k)}-\Phi\left(x^{(k)}\right)\right) \\
& -\lambda_{k}\left(x^{(k)}-x^{(0)}\right) .
\end{aligned}
$$

When $k \rightarrow \infty$, because $X\left(\lambda^{*}\right)$ and $y_{i}^{(k)}, i \notin I\left(x^{*}, \lambda^{*}\right)$, are bounded, the right-hand side of (23) is bounded. But, by assumption $\left(\mathrm{C}_{2}\right)$, if $y_{i}^{(k)} \rightarrow \infty, i \in I\left(x^{*}, \lambda^{*}\right)$, then the left-hand side of (23) tends to be infinite. This results in a contradiction.

(3) If $\lambda^{*}=0$, then the proof is similar to that of (12) because the nonempty index set $I_{0}\left(x^{*}, 0\right) \subset I\left(x^{*}, 0\right)$.

Now, we aim to prove our main result, that is, Theorem 7.

Theorem 7. Let $H$ be defined as in (7) and let assumptions $\left(C_{1}\right)-\left(C_{4}\right)$ hold. Then, for almost all $P^{(0)} \in X^{0}(1) \times R_{++}^{m} \times\{0\}$, there exists a $C^{1}$ curve $(P(s), \lambda(s))$ of dimension 1 such that

$$
\begin{aligned}
H\left(P(s), P^{(0)}, \lambda(s)\right) & =0, \\
(P(0), \lambda(0)) & =\left(P^{(0)}, 1\right) .
\end{aligned}
$$

When $\lambda(s) \rightarrow 0, P(s)$ tends to be point $P^{*}=\left(x^{*}, y^{*}, z^{*}\right)$, the $x$-component of which is a fixed point of $\Phi(x)$ in $X$.

Proof. By Lemmas 5 and 6, we obtain that there exists a $C^{1}$ curve $\Gamma_{P^{(0)}}$ starting from $\left(P^{(0)}, 1\right)$. For any $P^{(0)} \in X^{0}(1) \times R_{++}^{m} \times$ $R^{l}$, it is easy to show that $\partial_{P^{(0)}} H\left(P^{(0)}, 1\right) / \partial P$ is nonsingular. By Lemma 2, we conclude that $\Gamma_{P^{(0)}}$ is diffeomorphic to a unit interval.

Let $\left(P^{*}, \lambda^{*}\right)$ be a limit point of $\Gamma_{P^{(0)}}$, and then the following cases may occur:

(a) $\left(P^{*}, \lambda^{*}\right)=\left(x^{*}, y^{*}, z^{*}, \lambda^{*}\right) \in X \times R_{+}^{m} \times R^{l} \times\{0\}$.

(b) $\left(P^{*}, \lambda^{*}\right)=\left(x^{*}, y^{*}, z^{*}, \lambda^{*}\right) \in X^{0}(1) \times R_{++}^{m} \times R^{l} \times\{1\}$.

(c) $\left(P^{*}, \lambda^{*}\right)=\left(x^{*}, y^{*}, z^{*}, \lambda^{*}\right) \in \partial\left(X\left(\lambda^{*}\right) \times R_{+}^{m} \times R^{l}\right) \times(0,1]$.

When $\lambda=1$, the homotopy equation (7) becomes

$$
\begin{aligned}
& \sum_{j=1}^{l} \eta_{j}\left(x, z_{j}\right)+x-x^{(0)}=0, \\
& h(x)-\Theta h\left(x^{(0)}\right)=0, \\
& Y\left(g(x)-\Upsilon\left(g\left(x^{(0)}\right)+e_{m}\right)\right) \\
& \quad-Y^{(0)}\left(g\left(x^{(0)}\right)-\Upsilon\left(g\left(x^{(0)}\right)+e_{m}\right)\right)=0 .
\end{aligned}
$$

From (26) and (27), we obtain $x \in X^{0}(1)$. Then, assumption $\left(\mathrm{C}_{4}\right)$, together with (25), yields that $x=x^{(0)}$. By assumption $\left(\mathrm{C}_{2}\right)$, we obtain $z_{j}=0, j=1, \ldots, l$. Besides, it follows from (27) that $y=y^{(0)}$. Then, (25)-(27) have a unique solution $P=P^{(0)}=\left(x^{(0)}, y^{(0)}, 0\right)$ in $X^{0}(1) \times R_{++}^{m} \times R^{l} \times\{1\}$; therefore, case (b) is impossible. 
In case (c), we prove that $y^{*} \notin \partial R_{+}^{m}$. If $y^{*} \in \partial R_{+}^{m}$, then there exist $i_{0} \in\{1, \ldots, m\}$ and a sequence of points $\left\{\left(P^{(k)}, \lambda_{k}\right)\right\} \subset \Gamma_{P^{(0)}}$ such that $y_{i_{0}}^{(k)} \rightarrow y_{i_{0}}^{*}=0$ as $k \rightarrow+\infty$. From (14), we obtain

$$
\begin{aligned}
y_{i_{0}}^{(k)} & \left(g_{i_{0}}\left(x^{(k)}\right)-\lambda_{k} \gamma_{i_{0}}\left(g_{i_{0}}\left(x^{(0)}\right)+1\right)\right) \\
= & \lambda_{k} y_{i_{0}}^{(0)}\left(g_{i_{0}}\left(x^{(0)}\right)-\gamma_{i_{0}}\left(g_{i_{0}}\left(x^{(0)}\right)+1\right)\right) .
\end{aligned}
$$

When $k \rightarrow+\infty$, because $X(\lambda)$ and $(0,1]$ are bounded, the left-hand side of (28) tends to be 0 . Simultaneously, the righthand side of (28) tends to be $\lambda^{*} y_{i_{0}}^{(0)}\left(g_{i_{0}}\left(x^{(0)}\right)-\gamma_{i_{0}}\left(g_{i_{0}}\left(x^{(0)}\right)+\right.\right.$ $1)$ ), which is strictly less than 0 . This results in a contradiction.

Then, we prove that $x^{*} \notin \partial X\left(\lambda^{*}\right)$. If $x^{*} \in \partial X\left(\lambda^{*}\right)$, then there exist $i_{0} \in\{1, \ldots, m\}$ and a sequence of points $\left\{\left(P^{(k)}, \lambda_{k}\right)\right\} \subset \Gamma_{P^{(0)}}$ such that $g_{i_{0}}\left(x^{(k)}\right)-\lambda_{k} \gamma_{i_{0}}\left(g_{i_{0}}\left(x^{(0)}\right)+1\right) \rightarrow 0$, $y_{i_{0}}^{(k)} \rightarrow \infty$ as $k \rightarrow+\infty$. This contradicts Lemma 6; thus, case (c) is also impossible.

From the above discussion, we conclude that case (a) is the only possible case. Therefore, $P^{*}$ is a solution of (7) when $\lambda=0$, and $x^{*}$ is a fixed point of $\Phi(x)$ in $X$.

By differentiating the first equation in (24), we obtain Theorem 8, which, together with Theorem 7, can reduce various predictor-corrector algorithms (see [8] and the references therein).
Theorem 8. The homotopy path $\Gamma_{P^{(0)}}$ is determined by the following initial value problem to the ordinary differential equation:

$$
\begin{aligned}
D H(P(s), \lambda(s))\left(\begin{array}{c}
\dot{P}(s) \\
\dot{\lambda}(s)
\end{array}\right) & =0, \\
(P(0), \lambda(0)) & =\left(P^{(0)}, 1\right),
\end{aligned}
$$

where $s$ is the arc length of the curve $\Gamma_{P^{(0)}}$.

In implementing the predictor-corrector algorithm, we must proceed along the positive direction of the unit tangent vector $v$ at a point on $\Gamma_{P^{(0)}}$. The criterion that determines the positive direction is based on the condition that $v$ maintains the sign of the determinant of $\left(D H_{P^{(0)}}(P, \lambda)^{T}, v\right)^{T}$. In the first iteration, the sign is determined by the following lemma.

Lemma 9. If $\Gamma_{P^{(0)}}$ is smooth, then the positive direction $v^{(0)}$ at the initial guess $\left(P^{(0)}, 1\right)$ satisfies sign $\left|\begin{array}{c}D H_{P^{(0)}}\left(P^{(0)}, 1\right) \\ v^{(0)}{ }^{T}\end{array}\right|=$ $(-1)^{m+l+1}$.

Proof. Note that $D H_{P^{(0)}}\left(P^{(0)}, 1\right)=\partial H_{P^{(0)}}\left(P^{(0)}, 1\right) / \partial(P, \lambda)$ :

$\left.\begin{array}{cc}\nabla_{z}\left(\sum_{j=1}^{l} \eta_{j}\left(x^{(0)}, z_{j}^{(0)}\right)\right) & a^{(0)} \\ 0 & -\Theta h\left(x^{(0)}\right) \\ 0 & b^{(0)}\end{array}\right)=\left(M_{1}, M_{2}\right)$,

The tangent vector $v^{(0)}$ at $\left(P^{(0)}, 1\right)$ satisfies

$$
\left(M_{1}, M_{2}\right) v^{(0)}=\left(M_{1}, M_{2}\right)\left(\begin{array}{c}
v_{1}^{(0)} \\
v_{2}^{(0)}
\end{array}\right)=0
$$

where $v_{1}^{(0)} \in R^{n+m+l}$ and $v_{2}^{(0)} \in R^{1}$. From (32), we obtain $v_{1}^{(0)}=$ $-M_{1}^{-1} M_{2} v_{2}^{(0)}$.

Therefore, the determinant of $\left(\begin{array}{c}D H_{P^{(0)}\left(P^{(0)}, 1\right)} \\ v^{(0)^{T}}\end{array}\right)$ is 


$$
\begin{aligned}
& \left|\begin{array}{c}
D H_{P^{(0)}}\left(P^{(0)}, 1\right) \\
v^{(0)^{T}}
\end{array}\right|=\left|\begin{array}{cc}
M_{1} & M_{2} \\
v_{1}^{(0)^{T}} & v_{2}^{(0)^{T}}
\end{array}\right|=\left|\begin{array}{cc}
M_{1} & M_{2} \\
-M_{2}^{T} M_{1}^{-T} & 1
\end{array}\right| v_{2}^{(0)}=\left|M_{1}\right| v_{2}^{(0)}\left(1+M_{2}^{T} M_{1}^{-T} M_{1}^{-1} M_{2}\right) \\
& =\left|\begin{array}{ccc}
I & 0 & \nabla_{z}\left(\sum_{j=1}^{l} \eta_{j}\left(x^{(0)}, z_{j}^{(0)}\right)\right. \\
\nabla h\left(x^{(0)}\right)^{T} & 0 & 0 \\
Y^{(0)} \nabla g\left(x^{(0)}\right)^{T} \operatorname{diag}\left(g\left(x^{(0)}\right)-\lambda \Upsilon\left(g\left(x^{(0)}\right)+e_{m}\right)\right) & 0
\end{array}\right| \\
& \cdot v_{2}^{(0)}\left(1+M_{2}^{T} M_{1}^{-T} M_{1}^{-1} M_{2}\right) \\
& =|-I|\left|\operatorname{diag}\left(g\left(x^{(0)}\right)-\lambda \Upsilon\left(g\left(x^{(0)}\right)+e_{m}\right)\right)\right| v_{2}^{(0)}\left(1+M_{2}^{T} M_{1}^{-T} M_{1}^{-1} M_{2}\right) \\
& =(-1)^{l} \cdot\left|\operatorname{diag}\left(g\left(x^{(0)}\right)-\lambda \Upsilon\left(g\left(x^{(0)}\right)+e_{m}\right)\right)\right| \cdot v_{2}^{(0)}\left(1+M_{2}^{T} M_{1}^{-T} M_{1}^{-1} M_{2}\right) \text {. }
\end{aligned}
$$

Because $g\left(x^{(0)}\right)-\lambda \Upsilon\left(g\left(x^{(0)}\right)+e_{m}\right)<0,(1+$ $\left.M_{2}^{T} M_{1}^{-T} M_{1}^{-1} M_{2}\right)>0$, and $v_{2}^{(0)}>0$, the sign of the determinant $\left|\begin{array}{c}D H_{P^{(0)}}\left(P^{(0)}, 1\right) \\ v^{(0)^{T}}\end{array}\right|$ is $(-1)^{m+l+1}$.

\section{Numerical Results}

In this section, the numerical results provided below are obtained through the predictor-corrector algorithm. In each example, we set $\epsilon_{1}=1 \cdot e-3, \epsilon_{2}=1 \cdot e-6$, and $h_{0}=0.02$. The behavior of homotopy paths is graphically illustrated, which can deliver a visual insight into the performance of our computer code. Computational results are summarized in Table 1, where $x^{(0)}$ denotes the initial guess, IT is the number of iterations, $H$ is the value of $\left\|H_{P^{(0)}}\left(P^{(k)}, \lambda_{k}\right)\right\|$ when the algorithm stops, and $x^{*}$ is the fixed point.

Example 1. One finds a fixed point of self-mapping $\Phi(x)=$ $\left(x_{1}, 2-x_{2}\right)^{T}$ in

$$
\begin{aligned}
X & =\left\{\left(x_{1}, x_{2}\right) \in R^{2}: x_{1}-3 \leq 0,\left(x_{1}-2\right)^{2}\right. \\
& \left.+\left(x_{2}-1\right)^{2}-9=0\right\} .
\end{aligned}
$$

Let $\xi(x, y)=\nabla g(x) y$ and $\eta(x, z)=\left(24+2\left(x_{1}-2\right), 2\left(x_{2}-1\right)\right)^{T} z$, and then assumptions $\left(\mathrm{C}_{1}\right)-\left(\mathrm{C}_{4}\right)$ are satisfied. Based on the results of this study, we select initial points $x_{1}^{(0)}=(1,2)$ and $x_{2}^{(0)}=(-3,-2)$ which are not confined to the interior of the feasible sets. We obtain a fixed point $x^{*}=(-1,1)$ of $\Phi(x)$ in $X$ when $\lambda \rightarrow 0$, following the discrete homotopy pathways $\Gamma_{P^{(0)}}^{1}$ and $\Gamma_{P^{(0)}}^{2}$, as illustrated in Figure 1.
Example 2. One finds a fixed point of self-mapping $\Phi(x)=$ $\left(x_{1},-x_{2}\right)^{T}$ in the set

$$
\begin{aligned}
& X=\left\{\left(x_{1}, x_{2}\right) \in R^{2}:-x_{1}-x_{2}^{2}+8 \leq 0,3 x_{1}-2 x_{2}^{2}\right. \\
& -1=0\} .
\end{aligned}
$$

Let $\xi(x, y)=\nabla g(x) y$ and $\eta(x, z)=(6,0)^{T} z$, and then assumptions $\left(\mathrm{C}_{1}\right)-\left(\mathrm{C}_{4}\right)$ are satisfied. Based on the results of this study, we can select initial points $x_{3}^{(0)}=(-2,1)$ and $x_{4}^{(0)}=(-2,-1)$ which are not confined to the interior of the feasible sets. We obtain a fixed point $x^{*}=(0.333333,0)$ of $\Phi(x)$ in $X$ when $\lambda \rightarrow 0$, following the discrete homotopy pathways $\Gamma_{P^{(0)}}^{3}$ and $\Gamma_{P^{(0)}}^{4}$, as illustrated in Figure 2.

Example 3. One finds a fixed point of self-mapping $\Phi(x)=$ $\left(-x_{1}, x_{2}\right)^{T}$ in the set

$$
\begin{aligned}
X & =\left\{\left(x_{1}, x_{2}\right) \in R^{2}:-x_{1}-2 \pi \leq 0, x_{1}-2 \pi \leq 0, x_{2}\right. \\
& \left.-10 \cos \left(2 x_{1}\right)=0\right\} .
\end{aligned}
$$

Let $C^{2}$ functions $\xi(x, y)=\nabla g(x) y$ and $\eta(x, z)=(0,10)^{T} z$, and then assumptions $\left(C_{1}\right)-\left(C_{4}\right)$ are satisfied. Based on the results of this study, we can select initial points $x_{5}^{(0)}=(-3,3)$ and $x_{2}^{(0)}=(3,3)$ which are not confined to the interior of the feasible sets. We obtain a fixed point $x^{*}=(0,10)$ of $\Phi(x)$ in $X$ when $\lambda \rightarrow 0$, following the discrete homotopy pathways $\Gamma_{P^{(0)}}^{5}$ and $\Gamma_{P^{(0)}}^{6}$, as illustrated in Figure 3. 
TABLE 1: Numerical results of Examples 1-3.

\begin{tabular}{lcccccc}
\hline Examples & $x^{(0)}$ & IT & $\lambda^{*}$ & $H$ & $x^{*}$ & $\Phi\left(x^{*}\right)$ \\
\hline \multirow{2}{*}{ Example 1 } & $(1,2)$ & 5 & 0.000000 & 0.000000 & $(-1.000000,1.000002)$ & $(-1.000000,1.000002)$ \\
& $(-3,-2)$ & 8 & 0.000000 & 0.000000 & $(-1.000000,0.999999)$ & $(-1.000000,0.999999)$ \\
\hline \multirow{2}{*}{ Example 2 } & $(-2,1)$ & 7 & 0.000000 & 0.000000 & $(0.333333,0.000000)$ & $(0.333333,0.000000)$ \\
& $(-2,-1)$ & 8 & 0.000000 & 0.000000 & $(0.333333,-0.000000)$ & $(0.333333,-0.000000)$ \\
\hline \multirow{2}{*}{ Example 3 } & $(-3,3)$ & 11 & 0.000000 & 0.000000 & $(-0.000000,10.000000)$ & $(-0.000000,10.000000)$ \\
& $(3,3)$ & 11 & 0.000000 & 0.000000 & $(-0.000000,10.000000)$ & $(0.000000,10.000000)$ \\
\hline
\end{tabular}

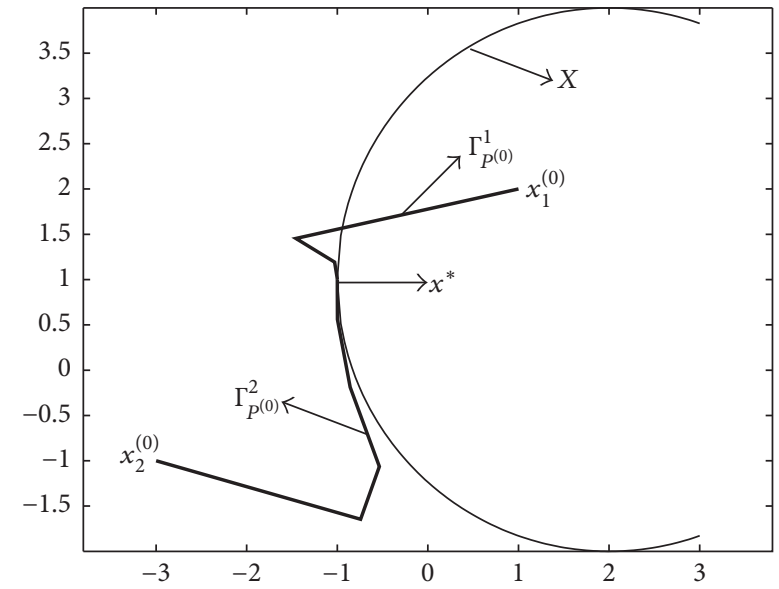

Figure 1: The discrete homotopy pathways of Example 1.

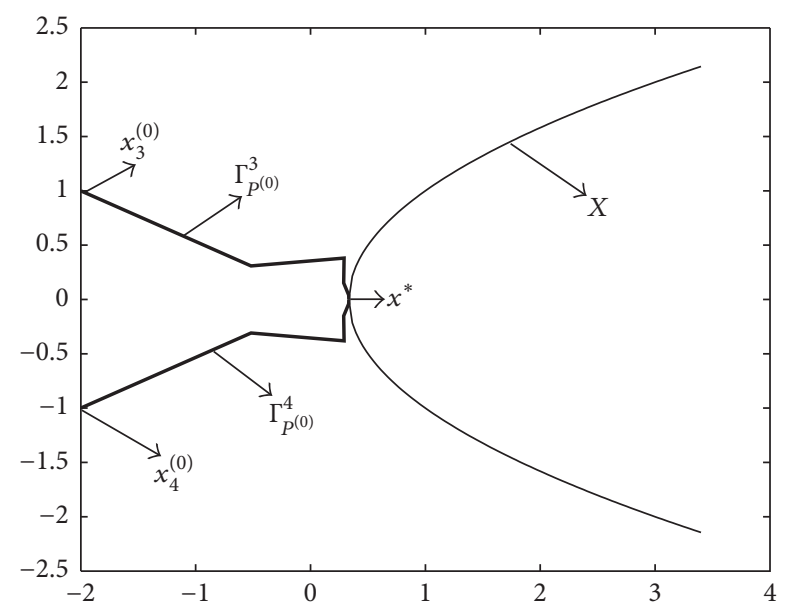

FIgURE 2: The discrete homotopy pathways of Example 2.

\section{Conflicts of Interest}

The authors declare that there are no conflicts of interest regarding the publication of this paper.

\section{Acknowledgments}

This work was supported by the National Natural Science Foundation of China (nos. 11671188 and U1304103), Innovation Scientists Technicians Troop Construction Projects of Henan Province (no. C20150027), and Innovation Scientists

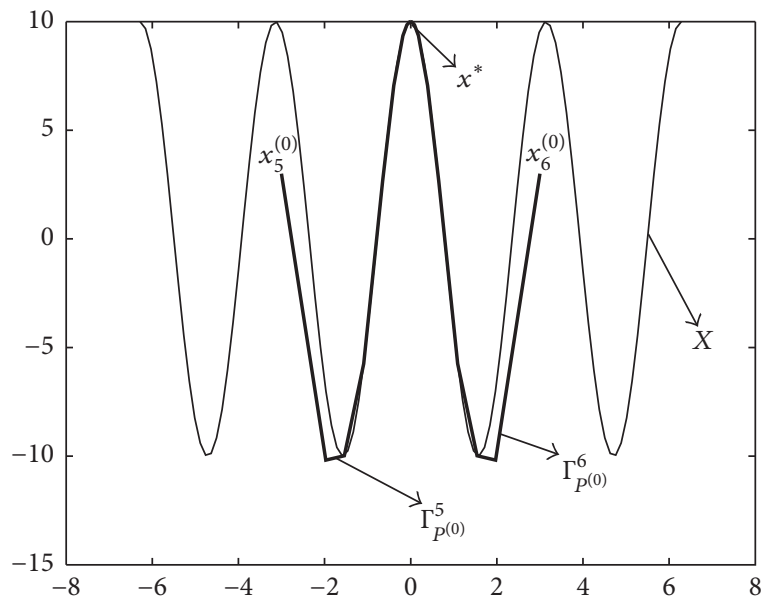

FIGURE 3: The discrete homotopy pathways of Example 3.

Technicians Troop Construction Projects of Luoyang Normal University (no. 2014-CXTD-001).

\section{References}

[1] B. Bollobas, W. Fulton, A. Katok, F. Kirwan, and P. Sarnak, Fixed Point Theory and Applications, Cambridge University Press, London, UK, 2004.

[2] S. Heikkila and K. Reffett, "Fixed point theorems and their applications to theory of Nash equilibria," Nonlinear Analysis. Theory, Methods \& Applications. An International Multidisciplinary Journal, vol. 64, no. 7, pp. 1415-1436, 2006.

[3] L.-J. Lin and Z.-T. Yu, "Fixed-point theorems and equilibrium problems," Nonlinear Analysis. Theory, Methods \& Applications. An International Multidisciplinary Journal, vol. 43, no. 8, pp. 987-999, 2001.

[4] S. Park, "Fixed points and quasi-equilibrium problems," Mathematical and Computer Modelling, vol. 32, no. 11-13, pp. 12971304, 2000.

[5] R. B. Kellogg, T. Y. Li, and J. Yorke, "A constructive proof of the Brouwer fixed-point theorem and computational results," SIAM Journal on Numerical Analysis, vol. 13, no. 4, pp. 473-483, 1976.

[6] S. N. Chow, J. Mallet-Paret, and J. A. Yorke, "Finding zeroes of maps: homotopy methods that are constructive with probability one," Mathematics of Computation, vol. 32, no. 143, pp. 887-899, 1978.

[7] J. C. Alexander and J. A. Yorke, "The homotopy continuation method: numerically implementable topological procedures," Transactions of the American Mathematical Society, vol. 242, pp. 271-284, 1978. 
[8] E. L. Allgower and K. Georg, Introduction to Numerical Continuation Methods, SIAM Society for Industried and Applied Mathematics, Philadelphia, Pa, USA, 2003.

[9] C. B. Carcia and W. I. Zangwill, "An approach to homotopy and degree theory," Mathematics of Operations Research, vol. 4, no. 4, pp. 390-405, 1979.

[10] Y. Li and Z. H. Lin, "A constructive proof of the Pincare-Birkhoff theorem," Transactions of the American Mathematical Society, vol. 347, no. 6, pp. 2111-2126, 1995.

[11] B. Yu and Z. Lin, "Homotopy method for a class of nonconvex Brouwer fixed-point problems," Applied Mathematics and Computation, vol. 74, no. 1, pp. 65-77, 1996.

[12] X. Fan and B. Yu, "A smoothing homotopy method for solving variational inequalities," Nonlinear Analysis. Theory, Methods \& Applications. An International Multidisciplinary Journal, vol. 70, no. 1, pp. 211-219, 2009.

[13] H. W. Li and Q. H. Liu, "A method to construct a quasi-normal cone for a class of complexity nonconvex sets and its applications in solving noncovex programming," Acta Mathematicae Applicatae Sinica, vol. 32, no. 3, pp. 400-412, 2009.

[14] H.-j. Xiong and B. Yu, "An aggregate deformation homotopy method for min-max-min problems with max-min constraints," Computational Optimization and Applications, vol. 47, no. 3, pp. 501-527, 2010.

[15] Y. Xiao, H. J. Xiong, and B. Yu, “Truncated aggregate homotopy method for nonconvex nonlinear programming," Optimization Methods \& Software, vol. 29, no. 1, pp. 160-176, 2014.

[16] Z. Zhu, B. Yu, and L. Yang, "Globally convergent homotopy method for designing piecewise linear deterministic contractual function," Journal of Industrial and Management Optimization, vol. 10, no. 3, pp. 717-741, 2014.

[17] Z. Zhou and B. Yu, "A smoothing homotopy method for variational inequality problems on polyhedral convex sets," Journal of Global Optimization, vol. 58, no. 1, pp. 151-168, 2014.

[18] M. Su and Z. Liu, "Modified homotopy methods to solve fixed points of self-mapping in a broader class of nonconvex sets," Applied Numerical Mathematics, vol. 58, no. 3, pp. 236-248, 2008.

[19] Z. Zhu, B. Yu, and Y. Shang, "A modified homotopy method for solving nonconvex fixed points problems," Fixed Point Theory and Applications, vol. 14, no. 2, pp. 531-544, 2013.

[20] M. Su, B. Yu, and S. Shi, "A boundary perturbation interior point homotopy method for solving fixed point problems," Journal of Mathematical Analysis and Applications, vol. 377, no. 2, pp. 683694, 2011.

[21] M. Su and X. Qian, "Existence of an interior path leading to the solution point of a class of fixed point problems," Journal of Inequalities and Applications, vol. 2015, no. 30, 2015.

[22] A. Capietto and F. Zanolin, "A continuation theorem for the periodic BVP in flow-invariant ENRs with applications," Journal of Differential Equations, vol. 83, no. 2, pp. 244-276, 1990. 


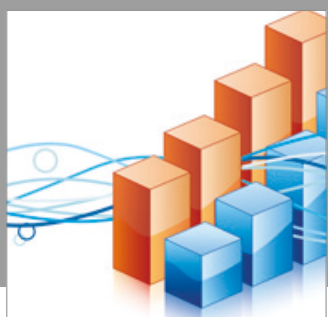

Advances in

Operations Research

vatersals

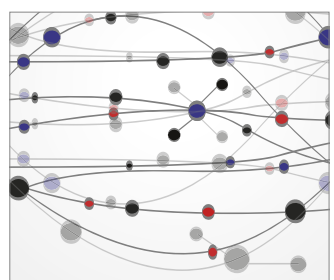

\section{The Scientific} World Journal
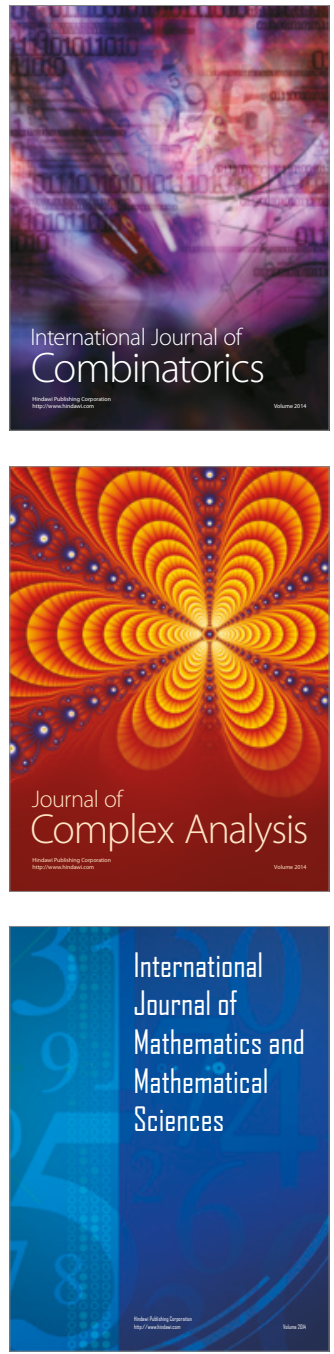
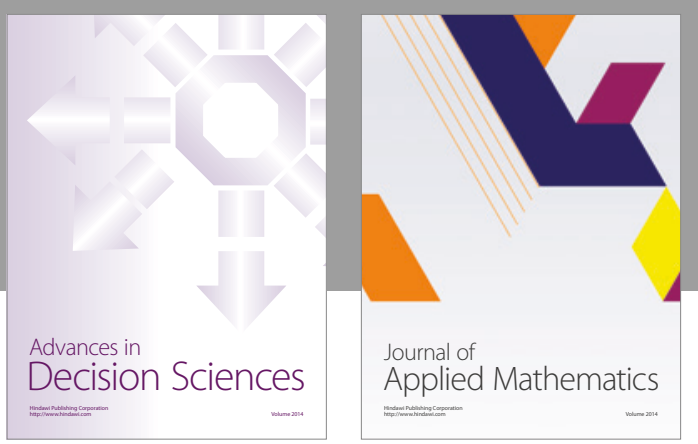

Algebra

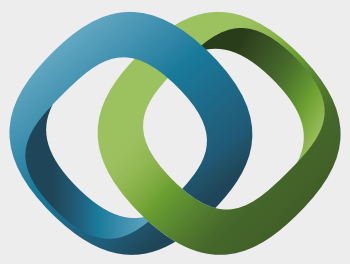

\section{Hindawi}

Submit your manuscripts at

https://www.hindawi.com
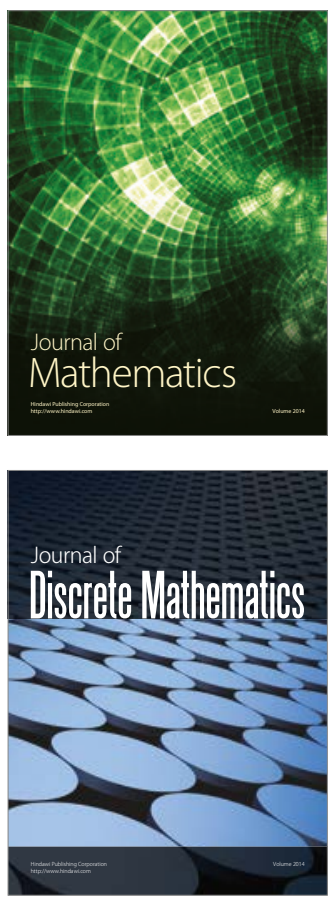

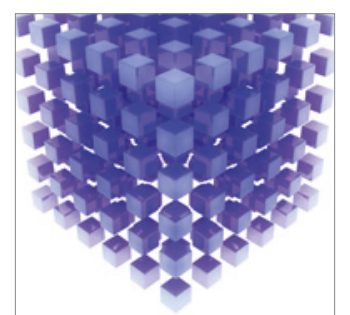

Mathematical Problems in Engineering
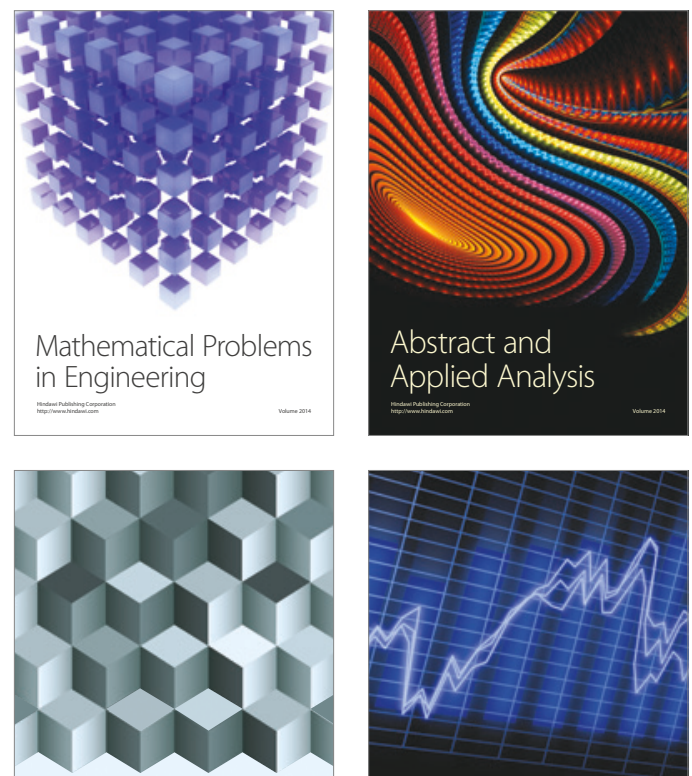

Journal of

Function Spaces

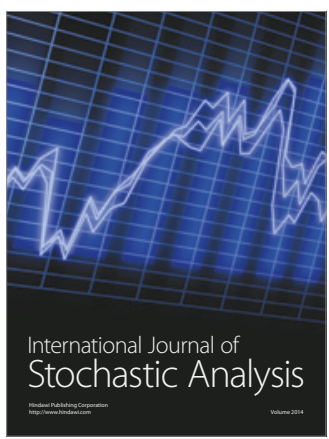

Probability and Statistics
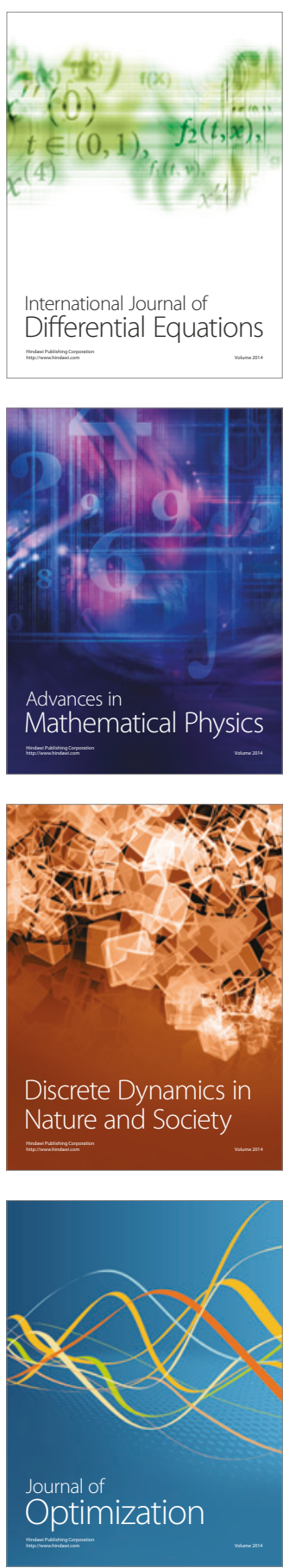